\title{
Magnetic-field and chemical-potential effects on the low-energy separation
}

\author{
J. M. P. Carmelo ${ }^{1}$ and A. H. Castro Neto ${ }^{2}$ \\ ${ }^{1}$ Department of Physics, University of Évora, Apartado 94, P-7001 Évora Codex, Portugal \\ and Centro de Física das Interacções Fundamentais, I.S.T., P-1096 Lisboa Codex, Portugal \\ ${ }^{2}$ Department of Physics, University of California, Riverside, CA 92521
}

(Received May 1996)

\begin{abstract}
We show that in the presence of a magnetic field the usual low-energy separation of the Hubbard chain is replaced by a " $c$ " and " $s$ " separation. Here $c$ and $s$ refer to small-momentum and low-energy independent excitation modes which couple both to charge and spin. Importantly, we find the exact generators of these excitations both in the electronic and pseudoparticle basis. In the limit of zero magnetic field these generators become the usual charge and spin fluctuation operators. The $c$ and $s$ elementary excitations are associated with the $c$ and $s$ pseudoparticles, respectively. We also study the separate pseudoparticle left and right conservation laws. In the presence of the magnetic field the small-momentum and low-energy excitations can be bosonized. However, the suitable bosonization corresponds to the $c$ and $s$ pseudoparticle modes and not to the usual charge and spin fluctuations. We evaluate exactly the commutator between the electronic-density operators. Its spin-dependent factor is in general non diagonal and depends on the interaction. The associate bosonic commutation relations characterize the present unconventional low-energy separation.
\end{abstract}

PACS numbers: 05.30. Jp, 05.30 Fk, 67.40. Db, 72.15. Nj

Typeset Using REVTEX 


\section{INTRODUCTION}

One of the central properties of one-dimensional many-electron problems [1] at zero magnetic field is the low-energy charge - spin separation [2]. This is related to the vanishing of the one-electron renormalization factor in these Luttinger liquids [3, 4]. However, in contrast to the case of the "g-ology" and Luttinger models [1, 2, 3], the study of the Bethe-ansatz (BA) solvable models [5,6, đ, 8] did not include a description of the low-energy physics in terms of operators with known expressions in the usual electronic basis: the Luttingerliquid character of these models relied on the identification of a structure in the low-energy spectrum provided by the BA [3,9].

Further, although there has been considerable progress in understanding the criticalenergy spectrum of multicomponent integrable systems in a magnetic field by combining BA and conformal-field theory [10], this approach also does not provide an operator description of the low-energy problem. A $c$ and $s$ pseudoparticle operator representation of such conformalfield theory of BA solvable models was introduced in Refs. [11,12. However, the expressions of the corresponding pseudoparticle generators in terms of the usual electronic operators remained an open question. Therefore, this latter study was unable to characterize the BA $c$ and $s$ excitation modes in terms of the usual charge or spin fluctuation operators.

In this paper we follow the preliminary results of Ref. [13] and derive the expressions of the low-energy and small-momentum $c$ and $s$ pseudoparticle generators [11,12 in terms of charge and spin electronic operators. This allows the description of these generators in terms of the usual charge and spin fluctuation operators for different values of the magnetic field and chemical potential. By writing such generators explicitly in the electronic basis we show that $c$ and $s$ are not in general charge and spin, respectively: for finite values of the magnetic field and chemical potential the $c$ and $s$ pseudoparticle-pseudohole modes couple

both to the charge and spin degrees of freedom. However, in the limits of zero magnetic field and zero chemical potential the $c$ and $s$ generators become the usual charge and spin fluctuation operators, respectively. 
The low-energy physics of the present many-electron problem can be described in terms of a Landau liquid of pseudoparticles. Such Landau-liquid approach was introduced in Refs. 14, 15, 16, 17, 18, 19. We show that this does not contradict its Luttinger-liquid character [3,13 which is explained by our pseudoparticle approach. We generalize for BA solvable many-electron problems the construction of the low-energy physics in terms of separate left and right conservation laws.

We also study the pseudoparticle $c$ and $s$ bosonic-operator algebra which describes the low-energy physics of integrable electronic Luttinger liquids in a magnetic field. In contrast to the bosonization scheme of the Luntinger model [1],3], the present bosonization refers to the many-electron ground state. In terms of the pseudoparticle interactions the latter has a non-interacting character - it is a simple Slatter determinant of pseudoparticle levels $111,13,20$.

References [16,18] include a study of the charge and spin fluctuations in terms of the pseudoparticle excitations. However, that study did not use an operator treatment of the problem. Therefore, it did not solve the present and inverse problem of expressing the $c$ and $s$ pseudoparticle excitation branches in terms of electronic charge and spin operators. This latter problem requires a careful analysis of the relevant Hilbert sub space where the charge and spin fluctuations are contained. This study is presented in the present paper in terms of the pseudoparticle operator generators of the Hamiltonian eigenstates.

Our operator analysis allows the solution of an important open problem. This is the evaluation of the commutator between the electronic-density operators for the many-electron Hubbard chain. This quantity plays an important role in the physics of many-electron systems [21]. We find the exact expression of this commutator for the Hubbard chain at finite values of the magnetic field and chemical potential. Its spin-dependent factor is in general non diagonal and depends on the interaction, electronic density, and spin density. This is the sign of the unconventional $c$ and $s$ separation which in the presence of the magnetic field replaces the usual charge and spin separation.

The paper is organized as follows: In section II we summarize some general features of 
the pseudoparticle basis. The generators of the $c$ and $s$ low-energy and small-momentum excitations are expressed both in the pseudoparticle and electronic basis in Sec. III. In section IV we explain the Luttinger-liquid character of the Hubbard chain by our pseudoparticle approach: we describe the low-energy physics in terms of separate left and right pseudoparticle conservation laws. The bosonization of the $c$ and $s$ pseudoparticle excitations and the evaluation of the commutator between the electronic-density operators are studied in Sec. V. Finally, Sec. VI contains the concluding remarks.

\section{THE PSEUDOPARTICLE OPERATOR BASIS}

In order to study the effects of the magnetic field on the generators of the low-energy and small-momentum excitations of the 1D integrable many-electron problem we consider in this paper the particular case of the Hubbard chain [7] in a magnetic field $H$ and chemical potential $\mu$ [11, 17, 18]

$$
\hat{H}=-t \sum_{j, \sigma}\left[c_{j \sigma}^{\dagger} c_{j+1 \sigma}+c_{j+1 \sigma}^{\dagger} c_{j \sigma}\right]+U \sum_{j}\left[c_{j \uparrow}^{\dagger} c_{j \uparrow}-\frac{1}{2}\right]\left[c_{j \downarrow}^{\dagger} c_{j \downarrow}-\frac{1}{2}\right]+2 \mu \hat{\eta}_{z}+2 \mu_{0} H \hat{S}_{z}
$$

where

$$
\hat{\eta}_{z}=-\frac{1}{2}\left[N_{a}-\sum_{\sigma} \hat{N}_{\sigma}\right], \quad \hat{S}_{z}=-\frac{1}{2} \sum_{\sigma} \sigma \hat{N}_{\sigma}
$$

Here $c_{j \sigma}^{\dagger}$ and $c_{j \sigma}$ are electron operators of spin projection $\sigma$ at site $j, \hat{N}_{\sigma}=\sum_{j} c_{j \sigma}^{\dagger} c_{j \sigma}$ and $t$, $U$, and $\mu_{0}$ are the transfer integral, the onsite Coulomb interaction, and the Bohr magneton, respectively. The Hamiltonian (1) describes $N_{\uparrow}$ up-spin and $N_{\downarrow}$ down-spin electrons in a lattice of $N_{a}$ sites $\left(N=N_{\uparrow}+N_{\downarrow}, n=N / N_{a}, n_{\sigma}=N_{\sigma} / N_{a}\right.$, and $\left.m=n_{\uparrow}-n_{\downarrow}\right)$. We consider in our study electronic densities $0<n<1$ and spin densities $0<m<n$. This corresponds

to a sector of parameter space with $U(1) \times U(1)$ symmetry [1],17,22, In this case the low-energy physics is dominated by the lowest-weight states (LWS's) of both the eta-spin and spin algebras which refer to real rapidities [11, 17, 22]. We call these states "LWS's I" to distinguish them from the LWS's associated with complex, non-real, rapidities, which we 
call "LWS's II". Importantly, in this $U(1) \times U(1)$ sector both the LWS's II and the nonLWS's have energy gaps relative to each canonical-ensemble ground state 20 and do not contribute to the low-energy physics [11,13.

In the pseudoparticle basis [11,13 one can define a many-pseudoparticle perturbation theory in which the non-interacting pseudoparticle ground state of simple Slater-determinant form is the exact ground state of the many-electron problem. All LWS's I can be generated by acting on the vacuum $|V\rangle$ (zero-electron state) with pseudoparticle operators. This operator algebra involves two types of anti-commuting pseudoparticle creation (annihilation) operators $b_{q \alpha}^{\dagger}\left(b_{q \alpha}\right)$ [11]. Here $\alpha$ refers to the two pseudoparticle colors $c$ and $s$ [11, 13, 18].

The discrete pseudomomentum values are $q_{j}=\frac{2 \pi}{N_{a}} I_{j}^{\alpha}$, where $I_{j}^{\alpha}$ are consecutive integers or half integers. There are $N_{\alpha}^{*}$ values of $I_{j}^{\alpha}$, i.e. $j=1, \ldots, N_{\alpha}^{*}$. One LWS I is specified by the distribution of $N_{\alpha}$ occupied values, which we call $\alpha$ pseudoparticles, over the $N_{\alpha}^{*}$ available values. There are $N_{\alpha}^{*}-N_{\alpha}$ corresponding empty values, which we call $\alpha$ pseudoholes. In the case of the Hubbard chain, we have that

$$
N_{c}^{*}=N_{a}, \quad N_{c}=N, \quad N_{s}^{*}=N_{\uparrow}, \quad N_{s}=N_{\downarrow},
$$

i.e., the pseudoparticle numbers are good quantum numbers. The boundary conditions fix the numbers $I_{j}^{\alpha}: I_{j}^{c}$ are integers (or half integers) for $N_{\downarrow}$ even (or odd), and $I_{j}^{s}$ are integers (or half integers) for $N_{\uparrow}$ odd (or even) [7, 11]. The ground state associated with a canonical ensemble of $\left(N_{\uparrow}, N_{\downarrow}\right)$ values [and $\left(N_{c}=N_{\uparrow}+N_{\downarrow}, N_{s}=N_{\downarrow}\right)$ ] has the form [1],13,20]

$$
\left|0 ; N_{\uparrow}, N_{\downarrow}\right\rangle=\prod_{\alpha=c, s}\left[\prod_{q=q_{F \alpha}^{(-)}}^{q_{F \alpha}^{(+)}} b_{q \alpha}^{\dagger}\right]|V\rangle .
$$

Here $b_{q \alpha}^{\dagger}$ is the pseudoparticle operator of pseudomomentum $q$ and of color $\alpha=c, s$. When $N_{\alpha}$ is odd (even) and $I_{j}^{\alpha}$ are integers (half integers) the pseudo-Fermi points are symmetric and given by $q_{F \alpha}^{(+)}=-q_{F \alpha}^{(-)}=\frac{\pi}{N_{a}}\left[N_{\alpha}-1\right]$. On the other hand, when $N_{\alpha}$ is odd (even) and $I_{j}^{\alpha}$ are half integers (integers) we have that $q_{F \alpha}^{(+)}=\frac{\pi}{N_{a}} N_{\alpha}$ and $-q_{F \alpha}^{(-)}=\frac{\pi}{N_{a}}\left[N_{\alpha}-2\right]$ or $q_{F \alpha}^{(+)}=\frac{\pi}{N_{a}}\left[N_{\alpha}-2\right]$ and $-q_{F \alpha}^{(-)}=\frac{\pi}{N_{a}} N_{\alpha}$

Except for terms of $1 / N_{a}$ order, $q_{F c}^{( \pm)}= \pm 2 k_{F}= \pm \pi n$ and $q_{F s}^{( \pm)}= \pm k_{F \downarrow}= \pm \pi n_{\downarrow}$. 
Let $\mathcal{H}_{\mathcal{I}}$ be the Hilbert space spanned by the LWS's I. In $\mathcal{H}_{\mathcal{I}}$ and normal-ordered relative to the ground state (4), the Hubbard Hamiltonian (1) has an infinite number of terms which correspond to increasing "order" of pseudoparticle scattering [11.[13]. In the Appendix we present such Hamiltonian and the associate pseudoparticle parameters.

\section{THE SMALL-MOMENTUM AND LOW-ENERGY $C$ AND $S$ GENERATORS}

Since the $c$ and $s$ pseudoparticles dominate the physics at energy scales smaller than the gaps for the LWS's II and non-LWS's, it is clearly of interest to study the low-energy and small-momentum generators in this region. The pseudoparticle operator algebra described in Refs. [11.12] is extracted directly from the BA solution. However, without the knowledge of the expressions of the Hamiltonian-eigenstate pseudoparticle generators in the electronic basis we cannot relate them to the usual charge and spin fluctuation operators. In this section we solve the problem for the case of the low-energy and small-momentum $c$ and $s$ generators which we express both in the pseudoparticle and electronic basis.

The usual charge and spin fluctuation operators read

$$
\hat{\rho}_{\rho}(k)=\hat{\rho}_{\uparrow}(k)+\hat{\rho}_{\downarrow}(k), \quad \hat{\rho}_{\sigma_{z}}(k)=\hat{\rho}_{\uparrow}(k)-\hat{\rho}_{\downarrow}(k),
$$

respectively, where

$$
\hat{\rho}_{\sigma}(k)=\sum_{k^{\prime}} c_{k^{\prime}+k \sigma}^{\dagger} c_{k^{\prime} \sigma}
$$

and $c_{k \sigma}^{\dagger}$ and $c_{k \sigma}$ are electron operators of spin projection $\sigma$ and momentum $k$.

Our goal is expressing the $\alpha=c, s$ pseudoparticle generators

$$
\hat{\rho}_{\alpha}(k)=\sum_{q} b_{q+k \alpha}^{\dagger} b_{q \alpha},
$$

in the electronic basis. Since the $\alpha$ pseudoparticle numbers are good quantum numbers of the many-electron problem [see Eq. (3)], the problem is solved at $k=0$. In this case the

operators (6) and (7) just provide the electronic and pseudoparticle number operators as follows 


$$
\hat{N}_{\sigma}=\hat{\rho}_{\sigma}(0), \quad \hat{N}_{\alpha}=\hat{\rho}_{\alpha}(0)
$$

respectively. The relation between the pseudoparticle and electronic numbers follows directly from Eq. (3) with the result

$$
\hat{N}_{\alpha}=\sum_{\sigma} G_{\alpha \sigma} \hat{N}_{\sigma}, \quad \hat{N}_{\sigma}=\sum_{\sigma} G_{\sigma \alpha}^{-1} \hat{N}_{\alpha}
$$

where the $\mathbf{G}$ and $\mathbf{G}^{-1}$ electron - pseudoparticle and pseudoparticle - electron charge matrices, respectively, read

$$
\mathbf{G}=\left[\begin{array}{ll}
G_{c \uparrow} & G_{c \downarrow} \\
G_{s \uparrow} & G_{s \downarrow}
\end{array}\right]=\left[\begin{array}{cc}
1 & 1 \\
0 & 1
\end{array}\right]=e^{\frac{1}{2} \sigma^{+}},
$$

and

$$
\mathbf{G}^{-1}=\left[\begin{array}{cc}
1 & -1 \\
0 & 1
\end{array}\right]=e^{-\frac{1}{2} \sigma^{+}}
$$

respectively, where $\sigma^{+}=\sigma_{x}+i \sigma_{y}$ is a Pauli matrix. The simple form of these matrices follows from the conservation of the number of $\alpha$ pseudoparticles. However, that $\hat{\rho}_{c}(0)=\hat{\rho}_{\rho}(0)$ and $\hat{\rho}_{s}(0)=\hat{\rho}_{\downarrow}(0)$ does not imply that such equalities hold true for $k>0$, as we find below.

The pseudoparticle representation can be extended to the whole Hilbert space. This requires the introduction of new pseudoparticle modes which are absent at low energy [23]. However, at low-energy there is a electron - pseudoparticle transformation which refers only to the present $c$ and $s$ pseudoparticle branches. An essential point is that this electron - pseudoparticle transformation does not mix left and right electronic operators, i.e., $\iota=$ $\operatorname{sgn}(k) 1= \pm 1$ electronic operators are made out of $\iota=\operatorname{sgn}(q) 1= \pm 1$ pseudoparticle operators only, $\iota$ defining the right $(\iota=1)$ and left $(\iota=-1)$ movers.

Measuring the electronic momentum $k$ and pseudomomentum $q$ from the $U=0$ Fermi points $k_{F \sigma}^{( \pm)}= \pm \pi n_{\sigma}$ and pseudo-Fermi points $q_{F \alpha}^{( \pm)}$, respectively, adds the index $\iota$ to the electronic and pseudoparticle operators. The new momentum $\tilde{k}$ and pseudomomentum $\tilde{q}$ are such that 


$$
\tilde{k}=k-k_{F \sigma}^{( \pm)}
$$

and

$$
\tilde{q}=q-q_{F \alpha}^{( \pm)}
$$

respectively, for $\iota= \pm 1$. For instance,

$$
\hat{\rho}_{\rho \iota}(k)=\hat{\rho}_{\uparrow \iota}(k)+\hat{\rho}_{\downarrow \iota}(k), \quad \hat{\rho}_{\sigma_{z \iota} \iota}(k)=\hat{\rho}_{\uparrow \iota}(k)-\hat{\rho}_{\downarrow \iota}(k),
$$

where

$$
\hat{\rho}_{\sigma \iota}(k)=\sum_{\tilde{k}} c_{\tilde{k}+k \sigma \iota}^{\dagger} c_{\tilde{k} \sigma \iota}
$$

Also

$$
\hat{\rho}_{\alpha \iota}(k)=\sum_{\tilde{q}} b_{\tilde{q}+k \alpha \iota}^{\dagger} b_{\tilde{q} \alpha \iota}
$$

In the Letter [13] we have expressed the pseudoparticle generators (7) in terms of the electronic operators (5) or (6) by restricting our analysis to a reduced Hilbert sub space. In order to justify the validity of that procedure we consider here the whole low-energy Hilbert space. Let us express the one-pair electronic operators (6) at $k=\iota \frac{2 \pi}{N_{a}}$ in the pseudoparticle basis. Henceforth we denote the ground state (4) by $|0\rangle$. The excitation

$$
\hat{\rho}_{\sigma \iota}\left(k=\iota \frac{2 \pi}{N_{a}}\right)|0\rangle
$$

is a superposition of both LWS's I, LWS's II, and non-LWS's. However, we are only interested in the low-energy component of this excitation. Therefore, we can omit here its LWS's II and non-LWS's components which refer to energy scales larger than the gaps of these states relative to the suitable ground state of form (4).

In references [11,20,22 we have constructed the low-energy Hamiltonian eigenstates by acting suitable pseudoparticle generators onto the pseudoparticle or pseudohole vacua. Following these studies, we can now describe the low-energy and momentum $k=\iota \frac{2 \pi}{N_{a}}$ Hilbert 
sub space where the excitation (17) is contained. This space is spanned by all Hamiltonian eigenstates of the form

$$
\left|\alpha \iota ; N_{p h}^{c}, N_{p h}^{s}, l\right\rangle=\hat{A}_{N_{p h}^{c}, N_{p h}^{s}, l}|\alpha \iota\rangle
$$

where $N_{p h}^{\alpha}$ is the number of $\alpha$ pseudoparticle-pseudohole processes such that $\frac{\sum_{\alpha} N_{p h}^{\alpha}}{N_{a}} \rightarrow 0$. In the right-hand side (rhs) of Eq. (18),

$$
|\alpha \iota\rangle=\hat{\rho}_{\alpha \iota}\left(k=\iota \frac{2 \pi}{N_{a}}\right)|0\rangle
$$

and

$$
\hat{A}_{N_{p h}^{c}, N_{p h}^{s}, l}=\prod_{\alpha=c, s} \hat{L}_{-N_{p h}^{\alpha \iota}}^{\alpha \iota}(l)
$$

where the operator $\hat{L}_{-N_{p h}^{\alpha \iota}}^{\alpha \iota}(l)$ is given in Eq. (56) of Ref. 12. It produces a number $N_{p h}^{\alpha, \iota}$ of $\alpha, \iota$ pseudoparticle-pseudohole processes. In the present case $\hat{A}_{N_{p h}^{c}, N_{p h}^{s}, l}$ is a zero-momentum operator and, therefore,

$$
N_{p h}^{\alpha}=\sum_{\iota} N_{p h}^{\alpha, \iota}=2 N_{p h}^{\alpha, \iota=1}=2 N_{p h}^{\alpha, \iota=-1}
$$

In equations (18) and (20) $l$ is a quantum number which distinguishes different pseudoparticle-pseudohole distributions characterized by the same values for the numbers (21). It follows from Eq. (21) that

$$
\sum_{\alpha, \iota} \iota N_{p h}^{\alpha, \iota}=0
$$

Since

$$
N_{p h}^{\alpha, \iota}=1,2,3, \ldots
$$

equations $(21)-(22)$ imply that

$$
\sum_{\alpha} N_{p h}^{\alpha}=\sum_{\alpha, \iota} N_{p h}^{\alpha, \iota}=2,4,6,8 \ldots
$$

is always an even positive integer. 
The set of Hamiltonian eigenstates of form (18) constitutes a complete and orthonormal basis which spans at low-energy the Hilbert space where the excitation (17) is defined. Therefore, at $k=\iota \frac{2 \pi}{N_{a}}$ it can be written as follows

$$
\hat{\rho}_{\sigma \iota}(k)|0\rangle=\sum_{\alpha, \iota, N_{p h}^{c}, N_{p h}^{s}, l}\left\langle\alpha \iota ; N_{p h}^{c}, N_{p h}^{s}, l\left|\hat{\rho}_{\sigma \iota}(k)\right| 0\right\rangle\left|\alpha \iota ; N_{p h}^{c}, N_{p h}^{s}, l\right\rangle, \quad k=\iota \frac{2 \pi}{N_{a}} .
$$

The methods used in Refs. [17, 19, 24] allowed us deriving the matrix elements of the rhs of Eq. (25). In the present $\frac{\sum_{\alpha} N_{p h}^{\alpha}}{N_{a}} \rightarrow 0$ limit which corresponds to the limit of vanishing excitation energy we find,

$$
\left\langle\alpha \iota ; N_{p h}^{c}, N_{p h}^{s}, l\left|\hat{\rho}_{\sigma \iota}\left(k=\iota \frac{2 \pi}{N_{a}}\right)\right| 0\right\rangle=0
$$

for $N_{p h}^{c}>0$ or (and) $N_{p h}^{s}>0$ and

$$
\left\langle\alpha \iota\left|\hat{\rho}_{\sigma \iota}\left(=\iota \frac{2 \pi}{N_{a}}\right)\right| 0\right\rangle=\sum_{\alpha^{\prime}} G_{\sigma \alpha^{\prime}}^{-1} \xi_{\alpha \alpha^{\prime}}^{1}
$$

where the matrix entries $G_{\sigma \alpha^{\prime}}^{-1}$ and $\xi_{\alpha \alpha^{\prime}}^{1}$ are defined in Eqs. (10) and (A4), respectively. While the finite matrix elements (27) can be derived from a spectral analysis of the charge and spin response functions [18], the result (26) follows from the method used in Ref. [19]. This is described for the particular case of one-electron matrix elements in Ref. [24].

We emphasize that the result (26) corresponds to vanishing excitation energy whereas at low but finite energy there is overlap between the excitation (17) and multi pseudoparticlepseudohole Hamiltonian eigenstates. Equations (26) and (27) tell us that in the limit of vanishing energy the excitation (17) only couples to the one-pair pseudoparticle-pseudohole Hamiltonian eigenstates of the form (19).

It is the vanishing-excitation-energy result $(26)$ - (27) which justifies the validity of the procedure followed in Ref. [13]: in order to find the expression of the $\alpha \iota$ pseudoparticle generators (16) we can consider only the reduced Hilbert space spanned by the four Hamiltonian eigenstates of form (19) and ignore all the multi-pseudoparticle-pseudohole states (18). The states (19) have momentum $k=\iota \frac{2 \pi}{N_{a}}$ relative to the ground state (4) and have a single pseudohole at one of the pseudo-Fermi points. Obviously, these states are orthogonal 
to that ground state. In the reduced Hilbert space they constitute a complete orthonormal basis, so that

$$
\left\langle\alpha \iota \mid \alpha^{\prime} \iota^{\prime}\right\rangle=\delta_{\alpha, \alpha^{\prime}} \delta_{\iota, \iota^{\prime}}
$$

and

$$
\sum_{\alpha, \iota}|\alpha \iota\rangle\langle\alpha \iota|=\mathbb{1}
$$

Using equations $(25)-(29)$ we can write $\hat{\rho}_{\sigma \iota}(k)$ in the reduced $k=\iota \frac{2 \pi}{N_{a}}$ Hilbert space with the result

$$
\hat{\rho}_{\sigma \iota}(k)=\sum_{\alpha, \iota} \mathcal{U}_{\alpha \sigma}^{-1} \hat{\rho}_{\alpha \iota}(k)
$$

where we have introduced the pseudoparticle - electronic matrix $\mathcal{U}^{-1}$ such that

$$
\mathcal{U}_{\alpha \sigma}^{-1}=\left\langle\alpha \iota\left|\hat{\rho}_{\sigma \iota}\left(k=\iota \frac{2 \pi}{N_{a}}\right)\right| 0\right\rangle
$$

Obviously, the result (30) holds true only at $k=\iota \frac{2 \pi}{N_{a}}$ and vanishing excitation energy.

Let us now introduce the "electronic" states

$$
|\sigma \iota\rangle=\hat{\rho}_{\sigma \iota}\left(k=\iota \frac{2 \pi}{N_{a}}\right)|0\rangle .
$$

Our task is finding the expression of the pseudoparticle operators $\hat{\rho}_{\alpha \iota}(k)$ in the electronic basis. Fortunately, the $k=\iota \frac{2 \pi}{N_{a}}$ states $|\sigma \iota\rangle$ constitute a complete (but non-orthonormal) basis in the reduced Hilbert space spanned by the four states of form (19). This holds true only in the corresponding limit of vanishing energy. At low but finite energy one finds out that the one-pair pseudoparticle operators (16) are both of one-pair and multipair electronic character. However, in the reduced Hilbert space we have that $\operatorname{det} \mathcal{U}^{-1}>0$ which implies that the set of four electronic states of form (32) constitutes a complete basis there. Therefore, we can invert the matrix $\mathcal{U}^{-1}$ and derive the following expression for the generator $(16)$

$$
\hat{\rho}_{\alpha \iota}(k)=\sum_{\sigma} \mathcal{U}_{\sigma \alpha} \hat{\rho}_{\sigma \iota}(k), \quad k=\iota \frac{2 \pi}{N_{a}},
$$


where the electronic - pseudoparticle matrix $\mathcal{U}$ is such that

$$
\mathcal{U}_{\sigma \alpha}=\sum_{\alpha^{\prime}} G_{\alpha^{\prime} \sigma} \xi_{\alpha \alpha^{\prime}}^{0}
$$

with the entries $G_{\alpha^{\prime} \sigma}$ and $\xi_{\alpha \alpha^{\prime}}^{0}$ defined in Eqs. (11) and (A4), respectively. We can alternatively express the pseudoparticle generators (16) in terms of the charge and spin fluctuation operators (14) with the result

$$
\hat{\rho}_{\alpha \iota}(k)=\mathcal{U}_{\rho \alpha} \hat{\rho}_{\rho \iota}(k)+\mathcal{U}_{\sigma_{z} \alpha} \hat{\rho}_{\sigma_{z} \iota}(k), \quad k=\iota \frac{2 \pi}{N_{a}},
$$

where

$$
\mathcal{U}_{\rho \alpha}=\frac{1}{2} \sum_{\alpha^{\prime}, \sigma= \pm 1} G_{\alpha^{\prime} \sigma} \xi_{\alpha \alpha^{\prime}}^{0}, \quad \mathcal{U}_{\sigma_{z} \alpha}=\frac{1}{2} \sum_{\alpha^{\prime}, \sigma= \pm 1} \sigma G_{\alpha^{\prime} \sigma} \xi_{\alpha \alpha^{\prime}}^{0}
$$

Equations (35) and (36) reveal that the generators of the $\alpha$ pseudoparticle excitations are an interaction-dependent mixture of charge and spin fluctuation operators. This holds true at finite values of the magnetic field and away of half filling where all the $2 \times 2$ matrix elements $\mathcal{U}_{\rho \alpha}$ and $\mathcal{U}_{\sigma_{z} \alpha}$ are non-vanishing and interaction dependent. This shows the exotic character of the $c-s$ low-energy separation.

However, from the usual $m=0$ and (or) $n=1$ pictures [2, 3],9], we expect $c$ and $s$ to become charge and spin, respectively, in the limit $m \rightarrow 0$, and $c$ to become charge in the limit $n \rightarrow 1$. This is confirmed by the data presented in the Table where we show some limiting forms of the pseudoparticle generators (35). We thus conclude that while at zero magnetic field there is the well known charge - spin separation of the low-energy and smallmomentum excitations of the Hubbard chain, the presence of the magnetic field changes the pre-existent charge and spin modes into new $c$ and $s$ separate excitation modes, respectively, which couple both to charge and spin. Our expression (35) fully characterizes the generators of these excitations in terms of the usual charge and spin fluctuation operators. 


\section{LOW-ENERGY PSEUDOPARTICLE LEFT AND RIGHT CONSERVATION LAWS}

Following the results of the Letter [13], the occurence at low-energy of separate right and left conservation laws [2] refers in BA solvable problems to the pseudoparticle scattering.

In this section we show that the Luttinger-liquid character of 1D interacting models solvable by BA follows from the properties of the Hamiltonian in the pseudoparticle operator basis. The pseudoparticle operator algebra is used to construct a general Luttinger-liquid theory. The non-interacting reference ground state of this theory is the Slatter determinant of pseudoparticle levels given in Eq. (4). This is the ground state of the many-electron problem. Moreover, the construction of the critical-point Hamiltonian proceeds by linearizing the pseudoparticle bands instead of the electronic bands.

Let us show that the pseudoparticle Hamiltonian (A1)-(A2) is the correct starting point to study the low-energy physics of the many-Hamiltonian (1) in terms of right and left conservation laws [2.13]. It also follows that in the presence of the magnetic field the lowenergy excitations can be bosonized. However, this bosonization corresponds to the $c$ and $s$ excitation modes studied in the previous section and refers to the pseudoparticle basis. In contrast to the zero-field case (where $c$ and $s$ are charge and spin, respectively - see the Table), the present bosonization scheme cannot be easily described in terms of charge and spin fluctuations. This follows from the exotic form of the generators (16) in terms of such fluctuations, Eq. (35).

While the two-electron amplitudes of scattering diverge at the Fermi points, the twopseudoparticle amplitudes of forward scattering and corresponding $f$ functions are finite and determine the low-energy parameters [11,13, 17, 24]. Therefore, the quantum problem (1) is non perturbative and perturbative in the electronic and pseudoparticle basis, respectively. This perturbative character of the pseudoparticle basis implies that the Hamiltonian (A1)(A3) can be used and is the more suitable as starting point for the construction of a criticalpoint Hamiltonian. 
This proceeds by linearizing the pseudoparticle bands $\epsilon_{\alpha}(q)$ around the pseudo-Fermi points and replacing the full $f$ functions by

$$
f_{\alpha \alpha^{\prime}}^{1}=f_{\alpha \alpha^{\prime}}\left(q_{F \alpha}^{( \pm)}, q_{F \alpha^{\prime}}^{( \pm)}\right)
$$

or

$$
f_{\alpha \alpha^{\prime}}^{-1}=f_{\alpha \alpha^{\prime}}\left(q_{F \alpha}^{( \pm)}, q_{F \alpha^{\prime}}^{(\mp)}\right)
$$

The expressions of $f_{\alpha \alpha^{\prime}}^{ \pm 1}$ are simple combinations of the pseudoparticle Landau parameters defined in Ref. [17 and involve the velocities $v_{\alpha}$ and parameters $\xi_{\alpha \alpha^{\prime}}^{j}$ of Eq. (A4) only. It is the form of this critical point Hamiltonian which implies the Luttinger-liquid character of the low-energy spectrum provided by BA and conformal-field theory [10]. The critical-point Hamiltonian can be written as

$$
: \hat{\mathcal{H}}:=\hat{\mathcal{H}}_{0}+\hat{\mathcal{H}}_{2}+\hat{\mathcal{H}}_{4}
$$

where

$$
\hat{\mathcal{H}}_{0}=\sum_{\alpha, \iota, \tilde{q}} \iota v_{\alpha} \tilde{q}: \hat{N}_{\alpha, \iota}(\tilde{q}):
$$

and

$$
\hat{\mathcal{H}}_{2}+\hat{\mathcal{H}}_{4}=\frac{2}{N_{a}} \sum_{\alpha, \alpha^{\prime}, \iota, \iota^{\prime}, k}\left[g_{2}^{\alpha \alpha^{\prime}}(k) \delta_{\iota,-\iota^{\prime}}+g_{4}^{\alpha \alpha^{\prime}}(k) \delta_{\iota, \iota^{\prime}}\right]: \hat{\rho}_{\alpha, \iota}(k):: \hat{\rho}_{\alpha^{\prime}, \iota^{\prime}}(-k):
$$

Here

$$
\hat{N}_{\alpha \iota}(\tilde{q})=b_{\tilde{q} \alpha \iota}^{\dagger} b_{\tilde{q} \alpha \iota}
$$

and the couplings read

$$
g_{2}^{\alpha \alpha^{\prime}}(k)=f_{\alpha \alpha^{\prime}}^{-1} \delta_{k, 0}
$$

and

$$
g_{4}^{\alpha \alpha^{\prime}}(k)=f_{\alpha \alpha^{\prime}}^{1} \delta_{k, 0}
$$


It follows that in the pseudoparticle basis the suitable critical-point Hamiltonian is a g-ology model of the type studied in Refs. [1,2] with exotic $k=0$ forward-scattering couplings, $\sigma$ replaced by $\alpha$, and the electronic operators by pseudoparticle operators.

This allows the ground-state distribution, $\left\langle\hat{N}_{\alpha}(q)\right\rangle$, to be equal to 1 inside and 0 outside the pseudo-Fermi surface [11, 17, 20], as confirmed by Eq. (4). The absence of the $g_{1}$ and $g_{3}$ terms implies that the $\alpha, \iota$ pseudoparticle number operators,

$$
\hat{N}_{\alpha, \iota}=\sum_{\tilde{q}} \hat{N}_{\alpha, \iota}(\tilde{q})
$$

are good quantum numbers, i.e. there are separate right and left conservation laws. This is a generalization of the results of Ref. [2] with the Fermi points replaced by the pseudo-Fermi points. In the case of single-component models [3, 25], we can omit the index $\alpha$, so that the BA critical Hamiltonian can be rewritten as

$$
: \hat{\mathcal{H}}:=\hat{\mathcal{H}}_{0}+\hat{V}
$$

with

$$
\hat{V}=\frac{\pi}{N_{a}} \sum_{\iota, \iota^{\prime}, k}\left[V_{1}(k) \delta_{\iota, \iota^{\prime}}+V_{2}(k) \delta_{\iota,-\iota^{\prime}}\right]: \hat{\rho}_{\iota}(k):: \hat{\rho}_{\iota^{\prime}}(-k):
$$

Here

$$
\begin{gathered}
\hat{\rho}_{\iota}(k)=\sum_{\tilde{q}} b_{\tilde{q}+k \iota}^{\dagger} b_{\tilde{q} \iota}, \\
V_{1}(k)=\frac{f^{1}}{2 \pi} \delta_{k, 0},
\end{gathered}
$$

and

$$
V_{2}(k)=\frac{f^{-1}}{2 \pi} \delta_{k, 0}
$$

Therefore, in single-component integrable systems, : $\hat{\mathcal{H}}:$ is a Luttinger model with exotic $k=0$ forward-scattering potentials. This justifies the Luttinger-liquid character of integrable 
models by BA. However, we have confirmed here that such character corresponds in the integrable many-electron problems to the pseudoparticle basis.

The separate right and left conservation laws provide the Luttinger-liquid parameters through equations of motions [2:13]. Let $\vartheta$ denote charge $(\vartheta=\rho)$ or $\operatorname{spin}\left(\vartheta=\sigma_{z}\right)$. Then $\hat{N}_{\vartheta \iota}$ can be written as

$$
\hat{N}_{\vartheta \iota}=\sum_{\alpha} k_{\vartheta \alpha} \hat{N}_{\alpha \iota}
$$

where the integers $k_{\vartheta \alpha}$ are $k_{\rho c}=k_{\sigma_{z} c}=1, k_{\rho s}=0$, and $k_{\sigma_{z} s}=-2 . k_{\uparrow s}=-1$.

Let us consider the charge or spin operator (14) which in the present notation are referred as $\hat{\rho}_{\vartheta \iota}(k)$. It is useful to consider the combinations

$$
\hat{\rho}_{\vartheta}^{( \pm)}(k)=\hat{\rho}_{\vartheta 1}(k) \pm \hat{\rho}_{\vartheta-1}(k)
$$

(note that $\left.\hat{\rho}_{\vartheta}^{(+)}(k)=\hat{\rho}_{\vartheta}(k)\right)$. Since the commutator

$$
\left[\hat{\rho}_{\vartheta}^{( \pm)}(k, t), \hat{\mathcal{H}}_{2}+\hat{\mathcal{H}}_{4}\right]=0
$$

for $k>0$ and

$$
\left[\hat{\rho}_{\vartheta}^{( \pm)}(k, t),: \hat{\mathcal{H}}:\right]
$$

is proportional to $k$ at $k=0$, the interesting quantity associated with the equation of motion for $\hat{\rho}_{\vartheta}^{( \pm)}(k, t)$ [2,13] is the following ratio

$$
\left.\frac{i \partial_{t} \hat{\rho}_{\vartheta}^{( \pm)}(k, t)}{k}\right|_{k=0}=\left.\frac{\left[\hat{\rho}_{\vartheta}^{( \pm)}(k, t),: \hat{\mathcal{H}}:\right]}{k}\right|_{k=0}=\mathcal{V}_{\vartheta}^{(\mp)} \hat{\rho}_{\vartheta}^{(\mp)}(0, t)
$$

where

$$
\mathcal{V}_{\vartheta}^{(-)}=\sum_{\alpha, \alpha^{\prime}} k_{\vartheta \alpha} k_{\vartheta \alpha^{\prime}}\left[v_{\alpha} \delta_{\alpha, \alpha^{\prime}}+\frac{f_{\alpha \alpha^{\prime}}^{1}-f_{\alpha \alpha^{\prime}}^{-1}}{2 \pi}\right]=\sum_{\alpha} v_{\alpha}\left[\sum_{\alpha^{\prime}} k_{\vartheta \alpha^{\prime}} \xi_{\alpha \alpha^{\prime}}^{1}\right]^{2}
$$

and

$$
\mathcal{V}_{\vartheta}^{(+)}=\frac{1}{\sum_{\alpha, \alpha^{\prime}} \frac{k_{\vartheta \alpha} k_{\vartheta \alpha^{\prime}}}{v_{\alpha} v \alpha^{\prime}}\left[v_{\alpha} \delta_{\alpha, \alpha^{\prime}}-\frac{A_{\alpha \alpha^{\prime}}^{1}+A_{\alpha \alpha^{\prime}}^{-1}}{2 \pi}\right]}=\frac{1}{\left\{\sum_{\alpha} \frac{1}{v_{\alpha}}\left[\sum_{\alpha^{\prime}} k_{\vartheta \alpha^{\prime}} \xi_{\alpha \alpha^{\prime}}^{1}\right]^{2}\right\}}
$$


Here $A_{\alpha \alpha^{\prime}}^{1}=A_{\alpha \alpha^{\prime}}\left(q_{F \alpha}^{( \pm)}, q_{F \alpha^{\prime}}^{( \pm)}\right)$and $A_{\alpha \alpha^{\prime}}^{-1}=A_{\alpha \alpha^{\prime}}\left(q_{F \alpha}^{( \pm)}, q_{F \alpha^{\prime}}^{(\mp)}\right)$, where $A_{\alpha \alpha^{\prime}}\left(q, q^{\prime}\right)$ are the scattering amplitudes given by Eqs. (83) - (85) of Ref. [18]. The velocities $\mathcal{V}_{\vartheta}^{(+)}$and $\mathcal{V}_{\vartheta}^{(-)}$determine the $\vartheta$ susceptibility, $K^{\vartheta}=1 /\left[\pi \mathcal{V}_{\vartheta}^{(+)}\right]$, and the coherent part of the $\vartheta$ conductivity spectrum, $\mathcal{V}_{\vartheta}^{(-)} \delta(\omega)$, respectively [2, 13]. This agrees with the studies of Refs. [17,18].

For single-component systems [25] there is only one choice for $\vartheta$ and $\mathcal{V}^{(-)}=v\left[\xi^{1}\right]^{2}$ and $\mathcal{V}^{(+)}=v\left[\xi^{0}\right]^{2}=v /\left[\xi^{1}\right]^{2}$, in agreement with Ref. [3]. The $\mathcal{V}_{\vartheta}^{( \pm)}$are the expressions for the charge and spin "velocities" of integrable multicomponent Luttinger liquids.

Equation (54) involves the commutator of the pseudoparticle-Hamiltonian : $\hat{\mathcal{H}}:$, Eq. (39), with an electronic operator and, therefore, the velocities $\mathcal{V}_{\vartheta}^{( \pm)}$do not have the same simple form as for the g-ology model of Ref. [2]. Importantly, except for single-component integrable models, $\mathcal{V}_{\vartheta}^{(+)}$does not equal the expression of $\mathcal{V}_{\vartheta}^{(-)}$with $f_{\alpha \alpha^{\prime}}^{1}-f_{\alpha \alpha^{\prime}}^{-1}$ replaced by $f_{\alpha \alpha^{\prime}}^{1}+f_{\alpha \alpha^{\prime}}^{-1}$

\section{PSEUDOPARTICLE BOSONIZATION}

The bosonization of the critical-point Hamiltonian (39) is straightforward and refers to the non-interacting pseudoparticle ground state (4). We find that

$$
\left[\hat{\rho}_{\alpha \iota}(k), \hat{\rho}_{\alpha^{\prime} \iota^{\prime}}\left(-k^{\prime}\right)\right]=\delta_{\alpha, \alpha^{\prime}} \delta_{\iota,,^{\prime}} \delta_{k, k^{\prime}}\left(\iota k \frac{N_{a}}{2 \pi}\right),
$$

and the $\alpha$ bosonic operators are given by

$$
a_{k \alpha}^{\dagger}=\sqrt{\frac{2 \pi}{N_{a}|k|}} \sum_{\iota} \theta(\iota k) \hat{\rho}_{\alpha \iota}(k),
$$

for $k>0$.

This bosonization reproduces the results of conformal-field theory [10,12]: the bosons (59) refer to the tower excitations of Ref. [12], whereas the HWS's [12] of the Virasoro Algebras [10] are the current and "charge" excitations [3]. The low-energy separation refers to the colors $\alpha$ studied in Sec. III for all parameter space. Therefore, in the presence of the magnetic field and chemical potential our boson modes do not correspond to charge and 
spin but to the $c$ and $s$ excitations of exotic generators given by Eq. (35). It follows that in the electronic basis and at $k=\iota \frac{2 \pi}{N_{a}}$ the operators (59) read

$$
a_{k \alpha}^{\dagger}=\sqrt{\frac{2 \pi}{N_{a}|k|}} \sum_{\iota} \theta(\iota k)\left[\mathcal{U}_{\rho \alpha} \hat{\rho}_{\rho \iota}(k)+\mathcal{U}_{\sigma_{z} \alpha} \hat{\rho}_{\sigma_{z} \iota}(k)\right],
$$

with $\mathcal{U}_{\rho \alpha}$ and $\mathcal{U}_{\sigma_{z} \alpha}$ defined in Eq. (36).

The commutator (58) involves the "pseudoparticle-density" operators. On the other hand, the evaluation of the commutation relations between the electronic-density operators (15) is a problem of high physical interest. The corresponding commutator has an important role in the physics of a many-electron quantum liquid [21]. The results we have obtained in previous sections allow the exact solution of this problem for the Hubbard chain in a magnetic field and chemical potential. In order to calculate these commutation relations between the electronic-density operators (15) we use Eqs. (30) and (58) to find

$$
\left[\hat{\rho}_{\sigma \iota}(k), \hat{\rho}_{\sigma^{\prime} \iota^{\prime}}\left(-k^{\prime}\right)\right]=\Delta_{\sigma, \sigma^{\prime}} \delta_{\iota, \iota^{\prime}} \delta_{k, k^{\prime}}\left(\iota k \frac{N_{a}}{2 \pi}\right) \text {. }
$$

This commutator is related to the electronic finite on-shell forward scattering phase shift referred in Ref. [21]. However, the matrix

$$
\Delta=\left[\begin{array}{cc}
\Delta_{\uparrow, \uparrow} & \Delta_{\uparrow, \downarrow} \\
\Delta_{\downarrow, \uparrow} & \Delta_{\downarrow, \downarrow}
\end{array}\right]
$$

has not been evaluated for the Hubbard chain. We find it is non-diagonal and interaction dependent. Its entries read

$$
\Delta_{\sigma, \sigma^{\prime}}=\sum_{\alpha} \mathcal{U}_{\alpha \sigma}^{-1} \mathcal{U}_{\alpha \sigma^{\prime}}^{-1}
$$

Referring directly to the above electronic phase shifts, it is natural that this matrix is also determined by the pseudoparticle forward-scattering interactions. Indeed, it can be expressed in terms of the simple combinations of two-pseudoparticle forward-scattering phase shifts, parameters (A4). The use of expression (27) which defines the quantity (31) leads to

$$
\Delta=\left[\begin{array}{cc}
\left(\xi_{c c}^{1}-\xi_{c s}^{1}\right)^{2}+\left(\xi_{s c}^{1}-\xi_{s s}^{1}\right)^{2} & \left(\xi_{c c}^{1}-\xi_{c s}^{1}\right) \xi_{c s}^{1}+\left(\xi_{s c}^{1}-\xi_{s s}^{1}\right) \xi_{s s}^{1} \\
\left(\xi_{c c}^{1}-\xi_{c s}^{1}\right) \xi_{c s}^{1}+\left(\xi_{s c}^{1}-\xi_{s s}^{1}\right) \xi_{s s}^{1} & \left(\xi_{c s}^{1}\right)^{2}+\left(\xi_{s s}^{1}\right)^{2}
\end{array}\right]
$$


where the $\xi_{\alpha \alpha^{\prime}}^{1}$ 's are the entries of the matrix

$$
\xi^{1}=\left[\begin{array}{ll}
\xi_{c c}^{1} & \xi_{c s}^{1} \\
\xi_{s c}^{1} & \xi_{s s}^{1}
\end{array}\right],
$$

which is such that

$$
\operatorname{det}[\Delta]=\left(\operatorname{det}\left[\xi^{1}\right]\right)^{2}
$$

The entries $\xi_{\alpha \alpha^{\prime}}^{1}$ are the above simple combinations of two-pseudoparticle zero-momentum forward-scattering phase shifts defined by Eq. (A4). Therefore, the electronic matrix (64) is fully determined by the corresponding two-pseudoparticle forward-scattering collisions. This confirms that there is a direct relation between the electronic and pseudoparticle forward scattering [24].

We have just evaluated exactly the quantity $\Delta_{\sigma, \sigma^{\prime}}$ of the commutator (61). Our expression (64) refers to electronic densities $0<n<1$ and spin densities $0<m<n$. In this case the Hamiltonian symmetry is $U(1) \otimes U(1)$. In order to prove the expected $S U(2)$ invariance of the matrix $\Delta$ at zero magnetic field [21] we should consider several directions in spin space. This study will be presented elsewhere. However, effects of the $S U(2)$ spin symmetry show up in the limit $H \rightarrow 0$ of our present expression.

The interaction, density, and spin-density dependence of (64) is of important physical meaning. It describes the dependence on these parameters of the finite electronic on-shell forward-scattering phase shift [21]. In order to study the matrix (64) in different limits we present in the Appendix the corresponding limiting values for the matrix (65). Based on the expressions of the Appendix we find that when $U \rightarrow 0$ for $\mu>W / 2$ (and thus $n<1$ ) and $H>0$ (and thus $m>0$ ) [W is the Mott-Hubbard gap] (64) reduces to

$$
\Delta_{\sigma, \sigma^{\prime}}=\delta_{\sigma, \sigma^{\prime}}
$$

Let us now consider the zero-magnetic-field and half-filling limits of expression (64). This study reveals properties which are a direct manifestation of the spin $S U(2)$ symmetry at 
$H=0$. For instance, that symmetry implies that in the limit $H \rightarrow 0$ and $\mu>W / 2$ the following form for the entries of (64)

$$
\begin{aligned}
\Delta_{\sigma, \sigma^{\prime}} & =\delta_{\sigma, \sigma^{\prime}}-\frac{[1-\operatorname{det}[\Delta]]}{2} \\
& =\delta_{\sigma, \sigma^{\prime}}-\frac{1}{2}\left[1-\frac{\left(\xi_{0}\right)^{2}}{2}\right],
\end{aligned}
$$

where the parameter $\xi_{0}$ was studied in Ref. [17]. We recall that for densities $0<n<1$ it changes from $\sqrt{2}$ for $U \rightarrow 0$, to 1 as $U \rightarrow \infty$. Therefore, $\operatorname{det}[\Delta]=\frac{\left(\xi_{0}\right)^{2}}{2}$ changes from 1 for $U \rightarrow 0$, to $\frac{1}{2}$ as $U \rightarrow \infty$. The fact that in the present limit the entries (68) decouple in the spin-dependent $\delta_{\sigma, \sigma^{\prime}}$ simple term and in a $\sigma, \sigma^{\prime}$ independent term follows from the zero-field $S U(2)$ spin symmetry. Note also that the latter term arises because of the removal by the electron-electron interaction of density spectral weight from low energy. This is associated with values $\operatorname{det}[\Delta]<1$ for $U>0$. Obviously, in the limit $U \rightarrow 0$ expression (68) reads

$$
\Delta_{\sigma, \sigma^{\prime}}=\delta_{\sigma, \sigma^{\prime}}
$$

Note that the $H=0 S U(2)$ spin symmetry has other effects on the $H \rightarrow 0$ limit. For instance, the $H \rightarrow 0, U \rightarrow 0$ and $U \rightarrow 0, H \rightarrow 0$ limits of $\xi_{\alpha \alpha^{\prime}}^{1}$ (see the Appendix) do not commute. However, we emphasize that this does not show up in the quantity (64) which has the same value in both these limits.

When $H \rightarrow 0$ and $U \rightarrow \infty$ for $\mu>W / 2$ we find

$$
\Delta_{\sigma, \sigma^{\prime}}=\delta_{\sigma, \sigma^{\prime}}-1 / 4
$$

At finite values of the magnetic field the entries of (64) have not the simple form (68). For instance, in the $H \rightarrow H_{c}$ fully polarized limit (the critical field for onset of full polarized ferromagnetism, $H_{c}$, is given by the rhs of Eq. (2) of Ref. [18]) the result is

$$
\Delta=\left[\begin{array}{cc}
1+\left(\eta_{0}-1\right)^{2} & \eta_{0}-1 \\
\eta_{0}-1 & 1
\end{array}\right]
$$

where $\eta_{0}=\frac{2}{\pi} \tan ^{-1}\left(\frac{\sin (\pi n)}{u}\right)$. 
When $\mu \rightarrow W / 2$ (half filling) the matrix (64) reads

$$
\Delta=\left[\begin{array}{cc}
\left(1-\xi_{c s}^{1}\right)^{2}+\left(\xi_{s s}^{1}\right)^{2} & \left(1-\xi_{c s}^{1}\right) \xi_{c s}^{1}-\left(\xi_{s s}^{1}\right)^{2} \\
\left(1-\xi_{c s}^{1}\right) \xi_{c s}^{1}-\left(\xi_{s s}^{1}\right)^{2} & \left(\xi_{c s}^{1}\right)^{2}+\left(\xi_{s s}^{1}\right)^{2}
\end{array}\right]
$$

If we take both the $\mu \rightarrow W / 2$ and $H \rightarrow 0$ limits we find

$$
\Delta_{\sigma, \sigma^{\prime}}=\delta_{\sigma, \sigma^{\prime}}-1 / 4
$$

If we consider the $\mu \rightarrow W / 2$ and $H \rightarrow H_{c}$ limits the result is

$$
\Delta=\left[\begin{array}{cc}
2 & -1 \\
-1 & 1
\end{array}\right]
$$

Finally, in the limit $\mu \rightarrow W / 2+4 t$ (and thus $n \rightarrow 0$, which implies zero-electron density) we find when $H>0$

$$
\Delta=\left[\begin{array}{cc}
2 & -1 \\
-1 & 1
\end{array}\right]
$$

whereas if we take first the limit $H \rightarrow 0$ and then $\mu \rightarrow W / 2+4 t$ we arrive to

$$
\Delta_{\sigma, \sigma^{\prime}}=\delta_{\sigma, \sigma^{\prime}}-1 / 4
$$

In this case the $H=0$ spin $S U(2)$ symmetry has a direct effect on the limiting values of (64) which are different in the limits $H \rightarrow 0, \mu \rightarrow W / 2+4 t$ and $\mu \rightarrow W / 2+4 t, H \rightarrow 0$.

\section{CONCLUDING REMARKS}

In this paper we have expressed the generators of the $c$ and $s$ low-energy and small momentum excitation modes of the Hubbard chain in the electronic basis. This has revealed that such eigenstate excitation branches are an interaction, density, and magnetization dependent mixture of the charge and spin fluctuation operators. As it is illustrated by the data of the Table, in the limit of zero magnetic field the $c$ and $s$ generators become the charge and spin fluctuation operators, respectively, and in the limit of half filling (zero chemical 
potential) the $c$ generator becomes the charge fluctuation operator. Following that Table, when we approach the limit $U \rightarrow 0$ at finite values of the magnetic field [colun (a) in the Table] and at zero magnetic field [colun (b) in the Table] the $c$ and $s$ generators become different operators. This effect of the magnetic field follows from the removal of the spin $S U(2)$ symmetry and was already detected in Ref. [18]. Note also that in the limits of zero electronic density and zero magnetization the $s$ generator becomes the down-spin generator (15), whereas in the former limit the $c$ generator becomes the charge fluctuation operator (14).

Our study has also revealed that the Landau-liquid character of the pseudoparticle representation does not contradict the Luttinger-liquid character of the BA energy spectrum. (Also the conformal character of the energy spectrum of electronic BA solvable models 10 follows from the form of the quantum problem Hamiltonian in the pseudoparticle basis [12,17].) The Landau-liquid character refers to the description of the low-energy physics in terms of pseudoparticles with only zero-momentum and zero-energy forward scattering interactions [11,17]. We have shown that the construction of the correct critical-point Hamiltonian follows from the Hamiltonian written in the Landau-liquid pseudoparticle representation. The exotic zero-momentum forward-scattering character of the $g$ couplings (43) and (44) and potentials (49) and (50) follows directly from the above Landau character of the quantum problem. These Luttinger-liquid parameters are fully determined by the values of the general Landau-liquid forward-scattering $f$ functions at the pseudo-Fermi points.

The obtained Luttinger-liquid theory and associate bosonization refers to the pseudoparticle non-interacting ground state (4). However, we emphasize that in the electronic basis this is the exact many-electron ground state. Importantly, our study has allowed the evaluation of the exact expression of the commutator between electronic-density operators for densities $n<1$ and spin densities $m>0$. We have found that this important quantity [21] is interaction dependent. In the present work we have not considered different directions in spin space at zero magnetic field. However, the study of that commutator in the limit $H \rightarrow 0$ has revealed interesting effects of the zero-field $S U(2)$ spin symmetry. 


\section{ACKNOWLEDGMENTS}

We thank D. K. Campbell and N. M. R. Peres for stimulating discussions. This research was supported by the ISI Foundation (Torino) under the EU Contract No. ERBCHRX CT920020. 


\section{APPENDIX A: THE MANY-PSEUDOPARTICLE HAMILTONIAN}

In the low-energy Hilbert sub space $\mathcal{H}_{\mathcal{I}}$ introduced in Sec. II and in normal-ordered relative to the ground state of form (4), the Hubbard Hamiltonian (1) reads [11,17]

$$
: \hat{H}:=\sum_{i=1}^{\infty} \hat{H}^{(i)}
$$

where the first two terms are

$$
\hat{H}^{(1)}=\sum_{q, \alpha} \epsilon_{\alpha}(q): \hat{N}_{\alpha}(q):
$$

and

$$
\hat{H}^{(2)}=\frac{1}{N_{a}} \sum_{q, \alpha} \sum_{q^{\prime}, \alpha^{\prime}} \frac{1}{2} f_{\alpha \alpha^{\prime}}\left(q, q^{\prime}\right): \hat{N}_{\alpha}(q):: \hat{N}_{\alpha^{\prime}}\left(q^{\prime}\right): .
$$

The expressions for the pseudoparticle bands, $\epsilon_{\alpha}(q)$, and of the "Landau" $f$ functions, $f_{\alpha \alpha^{\prime}}\left(q, q^{\prime}\right)$, are given in Refs. [15] and [17], respectively. The latter involve the velocities

$v_{\alpha}(q)=\frac{d \epsilon_{\alpha}(q)}{d q}$ and the two-pseudoparticle forward-scattering phase shifts $\Phi_{\alpha \alpha^{\prime}}\left(q, q^{\prime}\right)$. These are defined in Ref. [17]. In particular, the velocities $v_{\alpha} \equiv v_{\alpha}\left(q_{F \alpha}^{(+)}\right)$and the parameters

$$
\xi_{\alpha \alpha^{\prime}}^{j}=\delta_{\alpha \alpha^{\prime}}+\Phi_{\alpha \alpha^{\prime}}\left(q_{F \alpha}^{(+)}, q_{F \alpha^{\prime}}^{(+)}\right)+(-1)^{j} \Phi_{\alpha \alpha^{\prime}}\left(q_{F \alpha}^{(+)}, q_{F \alpha^{\prime}}^{(-)}\right), \quad j=0,1
$$

play a determining role at the critical point. $\left(\xi_{\alpha \alpha^{\prime}}^{1}\right.$ are the entries of the transpose of the dressed-charge matrix [10].)

It is useful for the studies of this paper to present here the different limiting values of the $\xi_{\alpha \alpha^{\prime}}^{1}$ parameters (A4) (below $W$ is the Mott-Hubbard gap and half filling is reached when $\mu \rightarrow W / 2):$

For $U \rightarrow 0$ when $\mu>W / 2$ and $H>0$

$$
\xi^{1}=\left[\begin{array}{ll}
1 & 0 \\
1 & 1
\end{array}\right] .
$$

For $H \rightarrow 0$ when $\mu>W / 2$ 


$$
\xi^{1}=\left[\begin{array}{cc}
\xi_{0} & \xi_{0} / 2 \\
0 & 1 / \sqrt{2}
\end{array}\right]
$$

For $H \rightarrow 0$ and $U \rightarrow 0$ when $\mu>W / 2$

$$
\xi^{1}=\left[\begin{array}{cc}
\sqrt{2} & 1 / \sqrt{2} \\
0 & 1 / \sqrt{2}
\end{array}\right] .
$$

For $H \rightarrow 0$ and $U \rightarrow \infty$ when $\mu>W / 2$

$$
\xi^{1}=\left[\begin{array}{cc}
1 & 1 / 2 \\
0 & 1 / \sqrt{2}
\end{array}\right] .
$$

For $H \rightarrow H_{c}$

$$
\xi^{1}=\left[\begin{array}{ll}
1 & 0 \\
\eta_{0} & 1
\end{array}\right]
$$

(where $\eta_{0}=\frac{2}{\pi} \tan ^{-1}\left(\frac{\sin (\pi n)}{u}\right)$.)

For $\mu \rightarrow W / 2$

$$
\xi^{1}=\left[\begin{array}{ll}
1 & \xi_{c s}^{1} \\
0 & \xi_{s s}^{1}
\end{array}\right] .
$$

For $\mu \rightarrow W / 2$ and $H \rightarrow 0$

$$
\xi=\left[\begin{array}{cc}
1 & 1 / 2 \\
0 & 1 / \sqrt{2}
\end{array}\right] .
$$

For $\mu \rightarrow W / 2$ and $H \rightarrow H_{c}$

$$
\xi^{1}=\left[\begin{array}{ll}
1 & 0 \\
0 & 1
\end{array}\right] .
$$

For $\mu \rightarrow W / 2+4 t$ when $H>0$

$$
\xi^{1}=\left[\begin{array}{ll}
1 & 0 \\
0 & 1
\end{array}\right] .
$$


For $H \rightarrow 0$ and $\mu \rightarrow W / 2+4 t$

$$
\xi^{1}=\left[\begin{array}{cc}
1 & 1 / 2 \\
0 & 1 / \sqrt{2}
\end{array}\right] .
$$

Further information on the pseudoparticle representation can be found, for example, in Refs. 11, 17, 18, 22, 


\section{REFERENCES}

1 See for example: I. E. Dzyaloshinskii and A. I. Larkin, Sov. Phys. JETP 38, 202 (1974); J. Sólyom, Adv. Phys. 28, 201 (1979).

${ }^{2}$ C. Di Castro and W. Metzner, Phys. Rev. Lett. 67, 3852 (1991); Walter Metzner and Carlo Di Castro, Phys. Rev. B 47, 16107 (1993), and references therein.

${ }^{3}$ F. D. M. Haldane, Phys. Rev. Lett. 45, 1358 (1980); J. Phys. C 14, 2585 (1981); Phys. Lett. 81A, 153 (1981).

${ }^{4}$ Philip W. Anderson, Phys. Rev. Lett. 64, 1839 (1990); 652306 (1990); P. W. Anderson and Y. Ren, in High Temperature Superconductivity, edited by K. S. Bedell, D. E. Meltzer, D. Pines, and J. R. Schrieffer (Addison-Wesley, Reading, MA, 1990).

5 This ansatz was introduced for the case of the isotropic Heisenberg chain by H. A. Bethe, Z. Phys. 71, 205 (1931).

${ }^{6}$ For one of the first generalizations of the Bethe ansatz to multicomponent systems see C. N. Yang, Phys. Rev. Lett. 19, 1312 (1967).

7 Elliott H. Lieb and F. Y. Wu, Phys. Rev. Lett. 20, 1445 (1968).

${ }^{8}$ For a modern and comprehensive discussion of these issues, see V. E. Korepin, N. M. Bogoliubov, and A. G. Izergin, Quantum Inverse Scattering Method and Correlation Functions (Cambridge University Press, 1993).

${ }^{9}$ H. J. Schulz, Phys. Rev. Lett. 64, 2831 (1990), and references therein.

${ }^{10}$ Holger Frahm and V. E. Korepin, Phys. Rev. B 42, 10553 (1990); ibid. 43, 5653 (1991); Masao Ogata, Tadao Sugiyama, and Hiroyuki Shiba, ibid. 43, 8401 (1991).

11 J. M. P. Carmelo and A. H. Castro Neto, Phys. Rev. Lett. 70, 1904 (1993); J. M. P. Carmelo, A. H. Castro Neto, and D. K. Campbell, Phys. Rev. B 50, 3667 (1994); in The physics and mathematical physics of the Hubbard model, edited by D. K. Campbell, D. 
Baeriswyl, J. M. P. Carmelo, F. Guinea, and E. Louis (Plenum, NATO ASI Series B, Vol. 343 New York, 1995), page 117.

12 J. M. P. Carmelo, A. H. Castro Neto, and D. K. Campbell, Phys. Rev. B 50, 3683 (1994).

13 J. M. P. Carmelo, A. H. Castro Neto, and D. K. Campbell, Phys. Rev. Lett. 73, 926 (1994); (E) ibid., 74, 3089 (1995).

14 J. Carmelo and A. A. Ovchinnikov, J. Phys.: Condens. Matter 3, 757 (1991).

15 J. Carmelo, P. Horsch, P. A. Bares, and A. A. Ovchinnikov, Phys. Rev. B 44, 9967 (1991).

16 J. M. P. Carmelo and P. Horsch, Phys. Rev. Lett. 68, 871 (1992).

17 J. M. P. Carmelo, P. Horsch, and A. A. Ovchinnikov, Phys. Rev. B 45, 7899 (1992).

18 J. M. P. Carmelo, P. Horsch, and A. A. Ovchinnikov, Phys. Rev. B 46, 14728 (1992).

19 J. M. P. Carmelo, P. Horsch, D. K. Campbell, and A. H. Castro Neto, Phys. Rev. B 48, 4200 (1993).

${ }^{20}$ J. M. P. Carmelo and N. M. R. Peres, Phys. Rev. B 51, 7481 (1995).

${ }^{21}$ P. W. Anderson and D. Khveshchenko, preprint (1995).

${ }^{22}$ J. M. P. Carmelo and N. M. R. Peres, Nucl. Phys. B 458 [FS], 579 (1996).

${ }^{23}$ J. M. P. Carmelo, N. M. R. Peres, and D. K. Campbell, preprint.

${ }^{24}$ J. M. P. Carmelo and A. H. Castro Neto, submitted to Phys. Rev. B.

${ }^{25}$ A. H. Castro Neto, H. Q. Lin, Y. -H. Chen, and J. M. P. Carmelo, Phys. Rev. B 50, 14 032 (1994). 


\section{TABLE}

$\begin{array}{lllllll} & U \rightarrow 0(a) & U \rightarrow 0(b) & n \rightarrow 0(c) & m \rightarrow 0(d) & n_{\downarrow} \rightarrow 0(e) & n \rightarrow 1(f) \\ \hat{\rho}_{c \iota}(k) & \hat{\rho}_{\uparrow \iota}(k) & \frac{\hat{\rho}_{\rho \iota}(k)}{\sqrt{2}} & \hat{\rho}_{\rho \iota}(k) & \xi_{c c}^{0} \hat{\rho}_{\rho \iota}(k) & \sum_{\sigma} \mathcal{U}_{\sigma c} \hat{\rho}_{\sigma \iota}(k) & \hat{\rho}_{\rho \iota}(k) \\ \hat{\rho}_{s \iota}(k) & \hat{\rho}_{\downarrow \iota}(k) & -\frac{\hat{\rho}_{\sigma z \iota}(k)}{\sqrt{2}} & \hat{\rho}_{\downarrow \iota}(k) & -\frac{\hat{\rho}_{\sigma z \iota}(k)}{\sqrt{2}} & \hat{\rho}_{\downarrow \iota}(k) & \sum_{\sigma} \mathcal{U}_{\sigma s} \hat{\rho}_{\sigma \iota}(k)\end{array}$

TABLE - Limiting values of the $c$ and $s$ generators (16) at $k=\iota \frac{2 \pi}{N_{a}}$ and for (a) $U \rightarrow 0$ when $0<n<1$ and $n_{\uparrow}>n_{\downarrow}$; (b) $U \rightarrow 0$ when $0<n<1$ and $n_{\uparrow}=n_{\downarrow}$; (c) $n \rightarrow 0$ when $n_{\uparrow}>n_{\downarrow}$ and $U>0$; (d) $m \rightarrow 0$ when $0<n<1$ and $U>0$; (e) $n_{\downarrow} \rightarrow 0$ when $n_{\downarrow}<n_{\uparrow}$ (here $\mathcal{U}_{\uparrow c}=1$ ); and (f) $n \rightarrow 1$ when $U>0$. 